\title{
Movimiento estudiantil chileno de 2011 y la lógica educacional detrás de su crítica al neoliberalismo'
}

\author{
Andrés Donoso Romo² \\ ORCID: 0000-0001-9712-877X
}

\section{Resumen}

Cuando muchas sociedades de la región continúan buscando la manera más adecuada de implementar las orientaciones de signo neoliberal promovidas por los grandes organismos financieros multilaterales, y cuando muchas de estas sociedades miran a Chile como ejemplo a seguir para conseguirlo, el movimiento estudiantil chileno de 2011 entrega algunos contrapuntos dignos de considerar. Como repara contundentemente la literatura especializada, este movimiento fue la primera gran crítica masiva a ese ordenamiento neoliberal que se ha venido implementando en el país desde la década de 1980. Sin embargo, como se podrá apreciar más detenidamente en este artículo, además de criticarse al ordenamiento neoliberal en la educación superior se esbozó una nueva lógica a partir de la cual se podría reorganizar el mundo universitario, una lógica donde la solidaridad, el bien común y la justicia social poseen un lugar destacado. Aquí, a partir de la revisión de las principales fuentes primarias y secundarias en la materia, se exponen los antecedentes que permitirán bosquejar, precisamente, esa nueva lógica desde la cual el estudiantado chileno criticó al ordenamiento neoliberal: primero se entregan algunos elementos sociohistóricos claves para entender la emergencia del movimiento, luego se describen en profundidad sus fases e hitos fundamentales, después se ahonda en sus principales demandas y, para finalizar, se analizan integralmente dichas exigencias para desprender la lógica estudiantil, identificada aquí como liberal, presente en el subtexto de su crítica al neoliberalismo.

\section{Palabras clave}

Movimiento estudiantil - Chile - Neoliberalismo - Liberalismo - Educación superior.

1- Agradezco al Proyecto ANID/FONDECYT, Concurso Regular, $N^{\circ} 1180506$ y al financiamiento otorgado por ANID/PIA/Fondos Basales para Centros de Excelencia FB0003.

2- Universidad de Playa Ancha, Valparaíso, Chile; Universidad de Chile, Santiago, Chile. Contactos: andres.donoso@upla.cl; andres.donoso@ ie.uchile.cl

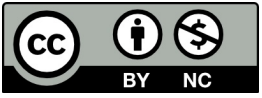




\section{The Chilean student movement of 2011 and the educational logic behind its criticism to neoliberalism}

\section{Abstract}

While several Latin American societies insist on searching for ways to implement neoliberal policies promoted by prominent International Financial Organizations, and while many of these societies point Chile as an example to follow, the Chilean Student Movement of 2011 has given us some counterpoints to consider. According to the evidence provided by specialized literature, the Chilean student movement of 2011 was the first massive criticism to neoliberalism in Chile since its introduction in the 1980s. However, in addition to the criticisms of neoliberalism, the students have pointed out the need to use a new logic to reorganize the university world. Which one? A logic where solidarity, common interest, and social justice are placed in a central position. Based on a methodology that included the review of the main primary and secondary sources, the article exposes the background that allows to know the critical aspects of this new logic advocated by the Chilean students. The paper is divided into five sections: firstly, some sociohistorical elements to understand the irruption of the movement are exposed, then its main phases and acknowledged milestones are described in depth, later its principal demands are studied and, finally, these demands are analyzed in order to infer the new logic advocated by the Student movement. This logic has been here provisionally named as "liberal".

\section{Keywords}

Student movement - Chile - Neoliberalism - Liberalism - Higher education.

\section{Introducción}

Treinta años de hegemonía neoliberal no pasan en vano. Desde el Golpe de Estado que en 1973 derrocó al gobierno de la Unidad Popular, y más aún desde que en 1980 el neoliberalismo comenzara a implementarse en las más diversas áreas del quehacer nacional, el centro político del país se viene desplazando hacia la derecha y, en ese mismo proceso, se viene modificando el sentido común de su población. Por esto, cuando el movimiento estudiantil de 2011 enarboló las banderas de la democratización de los gobiernos universitarios, del fin al lucro en las universidades y de la gratuidad en la educación superior, una parte importante de la población, en la cual me incluyo, quedó un tanto perpleja. Sorpresa que no se debía a que se desconocieran los graves problemas que afectaban a la educación en general, o a las universidades en particular, porque desde el momento mismo en que empezaron a sucederse las politicas neoliberales comenzaron también a acumularse los informes que subrayaban sus consecuencias negativas (GARCÍA 
MONGE; ISLA MADARIAGA; TORO BLANCO, 2006), se debía, más bien, a que las exigencias estudiantiles de 2011 se inscribían en una lógica que hasta entonces era prácticamente inimaginable. ¿Cuál lógica? Sobre ella es que trata este artículo.

Con base tanto en las sustanciosas descripciones existentes sobre el movimiento estudiantil de 2011, como en los profusos análisis sobre las implicancias que tuvo para el ordenamiento neoliberal, aquí se analizará un aspecto que pese a haber sido identificado como relevante por connotados investigadores, como Juan Eduardo García Huidobro y Alejandra Falabella (2013) y Sebastián Donoso-Díaz (2016), no ha sido examinado en profundidad, a saber, el trasfondo educacional de la protesta estudiantil. Conocimiento que permitirá llenar dicho vacío y, sobre todo, sopesar adecuadamente la identidad del movimiento. Ejercicio que permitirá contrarrestar algunas interpretaciones que invitan a entender que el actuar estudiantil se guiaba por idearios rupturistas, revolucionarios y/o insurreccionales. Interpretaciones defendidas, en su momento, tanto por manifestantes como por detractores que, pese a tener disímiles motivaciones, pues unos querían encauzar al movimiento mientras otros querían aislarlo, deseaban instalar la imagen de una movilización más radical de lo que en la práctica era.

Profundizar en el trasfondo educacional que tuvo el movimiento, en la dimensión propositiva por detrás de la crítica estudiantil, también permitirá aprehender la visión que tenían los manifestantes sobre el papel que debería asumir la educación en la conformación de sociedades solidarias o, al menos, más justas. Una preocupación que, por haber estado presente en todos los grandes movimientos estudiantiles de los últimos cien años en América Latina, permitirá poner en diálogo al movimiento chileno de 2011 con otros grandes movimientos de este tipo a nivel latinoamericano. Un ejercicio relevante, sobre todo, para aquellas personas interesadas en comprender a estos movimientos como fenómeno social y, más aún, para quienes poseen pretensiones comparativas ${ }^{3}$.

Para asir estos objetivos la investigación desplegó una metodología interdisciplinaria (DELLA PORTA; DIANI, 2015) con base histórica, que descansó en la revisión de documentos elaborados por los mismos estudiantes al calor de la movilización (manifiestos, convocatorias, cartas abiertas a autoridades $u$ otras) $y$ de materiales elaborados por analistas o protagonistas del mismo movimiento (artículos o libros). Las informaciones e interpretaciones obtenidas de estas fuentes, examinadas mediante un proceso sistemático de análisis de contenido (DRISKO; MASCHI, 2016), arrojó un conjunto de resultados que aquí se presentan organizados en cinco secciones. Primero se identifican las coordenadas históricas que enmarcaron al movimiento de 2011. Luego se describen las principales etapas e hitos del movimiento. Después se analizan sus demandas estructurales. Posteriormente, en la sección donde se expone el aporte original del artículo, se examina el trasfondo educacional que tuvo la crítica estudiantil al neoliberalismo. Y, finalmente, se despliegan los razonamientos que permiten concluir que el estudiantado ancló su cuestionamiento al ordenamiento neoliberal en un ideario de tipo democrático-liberal o, simplemente liberal, el mismo desde el cual desprendieron sus principales comprensiones educacionales.

3- La plataforma teórica que soporta las interpretaciones subsecuentes proviene de dos grandes matrices: los análisis sociopolíticos de la educación -llevados a cabo, entre otros, por Michael Apple (2013) - y los análisis sociohistóricos de los movimientos sociales -desarrollados, entre otros, por Charles Tilly y Sidney Tarrow (2015)-. 


\section{Coordenadas históricas del movimiento de 2011}

A diferencia de las decenas de golpes de Estado que azotaron a la región durante la segunda mitad del siglo XX, la asonada militar que ensombreció a Chile entre 1973 y 1990 no solo neutralizó a las fuerzas sociales que perseguían reestructurar la sociedad con miras a mejorar las condiciones de vida de los sectores populares, sino que también instaló un nuevo principio organizador de lo social: el neoliberalismo. Lógica que todavía pervive y que, con el paso de los años, fue imponiéndose también de manera más o menos disruptiva en la mayor parte de América Latina (GARRETÓN, 2012).

Este nuevo ordenamiento, en lo esencial, se ha caracterizado por limitar el rol del Estado en la conducción de la sociedad y por investir al Mercado como principal articulador de la misma. Orientaciones arraigadas en el entendimiento de que habían sido las políticas de promoción social impulsadas en las décadas pasadas por el Estado las culpables de los graves problemas que afectaban al país, en el supuesto de que las personas actuamos en lo social motivadas fundamentalmente por intereses individuales y en la convicción de que el desequilibrio económico entre los diversos sectores de la población no es una anomalía sino una condición necesaria para el buen funcionamiento de la sociedad (GARRETÓN, 2012). Con base en estos presupuestos, y luego de un turbulento período de ajuste estructural que coincidió con los momentos más duros de la así llamada Crisis de la deuda -vivida en toda América Latina en la década de 1980-, el neoliberalismo chileno consiguió algunos logros sustantivos, por ejemplo, controló la inflación, redujo el déficit fiscal, mejoró los indicadores de crecimiento económico y disminuyó el porcentaje de población en situación de pobreza (RIESCO, 2012). Todos éxitos que, sin embargo, se consiguieron a un alto precio: que se ignorara la cada vez más acentuada desigualdad económica y que se tolerara una democracia de baja intensidad donde esta situación no fuera cuestionada (RUIZ ENCINA, 2015).

Bajo estas coordenadas, desde 1980 el sistema educacional se ha venido reestructurando con el objetivo, más o menos explícito, de conformar un mercado donde primen los criterios económicos en las relaciones entre los sujetos, agentes y agencias educacionales. Escenario donde al Estado solo se le reserva un acotado papel: fomentar/ fiscalizar el accionar de los privados y proveer un mínimo de educación a quienes no poseen recursos suficientes para participar de este mercado. Una disposición, esta última, que alienta a las familias a desembolsar el máximo de dinero posible para alejar a los suyos de ese indeseado mínimo educacional. La observación de estas máximas ha conseguido, por un lado, que se profundice la secular división entre los circuitos educacionales reservados a los sectores dirigentes y a los demás sectores sociales -antes segmentados entre un adentro y un afuera del sistema educacional y ahora, con toda la población en edad escolar adentro del sistema, segregada en función de la capacidad de pago de los estudiantes- y ha logrado, por otro lado, que una proporción creciente de estudiantes se eduque en instituciones sometidas a criterios mercantiles, ergo, que se mercantilice el sistema educacional (BELLEI, 2015).

En la educación superior, puntualmente, el neoliberalismo viene implementándose desde la promulgación la Ley General de Universidades en 1981. Una legislación que en 
su momento se justificó como necesaria para frenar la politización creciente que se vivía al interior de las casas de estudio y para aliviar la fuerte presión que la educación superior ejercía sobre las arcas fiscales (SALAZAR; LEIHY, 2013). Gracias a este marco legal el país pasó de tener un sistema de educación superior basado en universidades estatales y privadas, ambas financiadas prácticamente en su totalidad por el Estado, a uno donde estos dos tipos de universidades -llamadas ahora tradicionales- solo son sustentadas parcialmente con recursos públicos y donde empiezan a proliferar instituciones íntegramente privadas. Cambios que redundan en que los estudiantes y sus familias financien el grueso de la educación superior: cerca del 80\% en las universidades que mantienen apoyo estatal -las tradicionales-, y el 100\% en aquellas integramente privadas. Siendo oportuno recordar que poco antes del conflicto de 2011, conforme informaron la Organización para la Cooperación y el Desarrollo Económico y el Banco Mundial, Chile era uno de los países donde más se invertía en educación superior, donde más exiguamente el Estado contribuía a su financiamiento y donde más altos eran los aranceles cobrados por las universidades en proporción a los ingresos per cápita (OCDE, 2009).

Cabe subrayar que en todas estas décadas el estudiantado universitario ha venido aumentando sostenidamente. Una evolución, no obstante, que experimentó un expresivo paréntesis durante la dictadura. Si en 1960 había 26 mil estudiantes universitarios, en 1967 eran 56 mil y en 1974 ascendian a 143 mil (BRUNNER, 2015). En 1990, luego del paréntesis dictatorial, el número de universitarios era de $131 \mathrm{mil}$, en 2000 llega a 319 mil y en 2010, un año antes del movimiento analizado, aumenta a 660 mil (SALAZAR; LEILHY, 2017). Este fuerte incremento de la matrícula universitaria, sumado a las políticas neoliberales implementadas desde 1980, trajeron consigo dos novedades. Por un lado, cada vez más jóvenes de los sectores medios y populares accedieron a la educación superior, lo que devino en que se fuera segmentando el sistema/mercado universitario en función de la capacidad de pago del estudiantado. Y, por otro lado, una parte importante de estos nuevos estudiantes ingresó a aquellas universidades que discriminaban únicamente por criterios económicos, lo que llevó a muchos a endeudarse para poder solventar sus estudios (SALAZAR; LAIHY, 2017).

El estudiantado chileno, con una historia de organización y movilización que se remonta a principios del siglo XX, no fue indiferente a estos procesos. A finales de la década de 1970, después del reflujo que produjo el Golpe de 1973, se rearticuló alrededor de asuntos deportivos y culturales y luego, en la década de 1980, acompañó activamente las protestas populares contra la dictadura. En 1987, cabe puntualizar, se registra el primer gran movimiento estudiantil en protesta por las graves carencias económicas que afectaban a la Universidad de Chile en particular y al sistema universitario en general (SALAZAR; PINTO, 2014). Durante la década de 1990, con especial fuerza en 1997, continuaron las movilizaciones estudiantiles por motivos financieros (THIELEMANN, 2016). No obstante, fue solo a comienzos del siglo XXI que estalló el primer gran movimiento social vivido en democracia, el que en 2006 lideraron los estudiantes secundarios en contra de la segmentación del sistema educacional y, más específicamente, contra las graves falencias en materia de calidad que presentaban las instituciones que acogían a los sectores populares (DONOSO, 2013). Todos antecedentes que dan cuenta que el movimiento de 
2011 no fue un caso aislado, al contrario, fue expresión de un descontento que se venía acumulando hace décadas.

\section{El movimiento estudiantil de 2011: fases e hitos}

El movimiento estudiantil de 2011 se prolongó prácticamente por todo ese año, abarcó al estudiantado de todas las universidades del país y se alzó contra uno de los rasgos estructurales del ordenamiento neoliberal: el lucro en las iniciativas educacionales. Siendo un movimiento, por tanto, notoriamente más prolongado, masivo e incisivo que sus antecesores. ¿Cómo entender esta particularidad? Probablemente porque por primera vez, desde que en 1990 se recupera la democracia, había un gobierno que se sentía plenamente cómodo con el ordenamiento neoliberal, lo que transformaba al ejecutivo en un antagonista para todo aquel que discrepara con el rumbo del país. Quizá, también, porque parte importante de los manifestantes de 2011 había participado del gran movimiento de 2006, lo cual, entre otros aspectos, hizo que se desarrollara una suerte de escepticismo militante que terminaría radicalizando al movimiento. Y posiblemente, a su vez, porque en 2011 el estudiantado comenzaba a cargar con las consecuencias de una de las políticas más refinadas del neoliberalismo educacional nacional: el Crédito con Aval del Estado. Política que en lo medular permitía que los bancos comerciales prestaran dinero a los universitarios. Iniciativa que fue evaluada por los manifestantes como un excelente negocio para las instituciones financieras, también para las universidades íntegramente privadas, y como un pésimo negocio tanto para el Estado como para los estudiantes que se acogían a este beneficio.

El peso que tuvieron estos antecedentes se fue haciendo patente desde la primera etapa del movimiento, experimentada entre marzo y mayo de 2011: el ascenso. Durante estos meses el estudiantado universitario protestó por agravios puntuales, como algunas dificultades asociadas a la asignación de becas de alimentación o posibles pérdidas de beneficios en materia de transporte escolar, y también por asuntos más complejos como la oscura venta de una universidad privada, la Universidad Central. Afrentas que hicieron que a fines de abril se verificaran las primeras movilizaciones, con varios miles de manifestantes. Protestas que paulatinamente fueron aumentando tanto en frecuencia como en masividad (FIGUEROA, 2013).

Durante esta primera fase de la movilización el gobierno reaccionó como generalmente lo venía haciendo ante este tipo de presiones, ignoró durante el mayor tiempo posible a los manifestantes y, cuando esto se tornó insostenible, intentó deslegitimarlos tachándolos de grupos minoritarios que no representaban el verdadero sentir de la juventud estudiosa ${ }^{4}$. Los manifestantes, reaccionado ante esta actitud de las autoridades -sobre todo después de que el presidente invisibilizara por completo sus exigencias en su cuenta pública anual ese 21 de mayo-, se propusieron redoblar sus protestas para así obligar al ejecutivo a que recapacitara (REYES; VALLEJO, 2013). Para fines de mayo, por tanto, el carácter de antagonista que adquirió finalmente el gobierno estaba plenamente establecido.

4- Ver, por ejemplo, las declaraciones del entonces Ministro de Educación en los primeros meses del conflicto (LAVÍn..., 2011). 
La segunda etapa del movimiento, su apogeo, se prolongó entre junio y agosto de ese año. Período donde el estudiantado desplegó una serie de medidas de presión que incluyó desde las más tradicionales, como los paros de actividades, las tomas de establecimientos educacionales, las concentraciones masivas o las marchas multitudinarias, hasta algunas más creativas como los bailes colectivos o las pruebas deportivas. Con el correr de las semanas las marchas de los jueves se fueron haciendo cada vez más concurridas y superaron en varias oportunidades los doscientos mil manifestantes -solo en Santiago-. Siendo en esta fase que se verificó la única jornada de protesta popular desde que en 1990 se retorna a la democracia -la que ese 4 de agosto transformó a la capital en un verdadero campo de batalla (FAURÉ; MIRANDA, 2016) - y que se celebró la protesta que a la postre sería la más masiva del movimiento -la concentración que el 28 de agosto congregó a cerca de un millón de personas en el Parque 0'Higgins-.

Fue en estos meses de apogeo del movimiento que el estudiantado universitario consiguió aglutinar tras de sí a diversos actores sociales vinculados a la educación. Fue entonces cuando se sumaron, al decir de Manuel Riesco, "los jóvenes de todas las edades" (2012, p. 252). Por esto es que al lado de los universitarios pudieron verse, además de sus familiares, muchos estudiantes secundarios, importantes sectores del profesorado primario, secundario y universitario, también muchos profesionales jóvenes y, asimismo, varios integrantes del mundo artístico e intelectual del país. Destacándose que por primera vez se plegaron a un movimiento de este tipo amplios contingentes estudiantiles de instituciones integramente privadas y subrayándose que pese a la diversidad de participantes no se evidenciaron grandes escisiones o pugnas al interior del movimiento (lo cual coincide con que tampoco se advirtió una disidencia estudiantil organizada en contra de las movilizaciones).

Quien en esta segunda etapa sí se posicionó frontalmente en contra de la juventud movilizada fue el gobierno, porque cuando no pudo seguir subestimando a los estudiantes levantó propuestas que, en vez de atender las tres grandes demandas del mundo estudiantil (participación, gratuidad y fin al lucro), solo aumentaban escuetamente el presupuesto público destinado a la educación superior. Una disposición que el ejecutivo mantendría sin grandes variaciones hasta el final del conflicto y que fue complementada con el despliegue de una doble estrategia de criminalización y hostigamiento hacia los manifestantes. Estrategia materializada en la detención masiva de estudiantes e, inclusive, en el asesinato de un joven, Manuel Gutiérrez, producto de una bala perdida disparada por un policía.

La última etapa del movimiento, su declive, se prolongó entre septiembre y diciembre de 2011. Meses en los cuales el estudiantado se desmovilizó de manera desordenada y sin conseguir ninguna respuesta satisfactoria a sus reclamos. Aun cuando no existe consenso sobre las razones detrás del desenlace, sí hay un cierto grado de acuerdo en la identificación de los factores que incidieron: a) a principios de septiembre el estudiantado perdió la tribuna que hasta entonces le había prodigado la prensa, pues ésta se volcó a cubrir la muerte traumática de un equipo de la televisión local; b) también desde septiembre comenzaron a ponerse en práctica una serie de negociaciones entre estudiantes y gobierno que, aun cuando no fueron efectivas, incidieron en que una parte de los manifestantes retornara 
a sus actividades habituales al percibir que se abría una vía de solución al conflicto; y c) en septiembre, igualmente, las autoridades ministeriales y universitarias comenzaron a presionar/amenazar sosteniendo que si persistía la movilización se perderian beneficios educacionales o, inclusive, el año académico.

\section{Las demandas estudiantiles}

Al momento de apreciar las demandas de ese 2011 lo primero que sobresale es su diversidad. Una cualidad, cabe reparar, propia de todo movimiento social que logra aglutinar tras de sí a grupos heterogéneos de personas. Las exigencias, como ilustra un reconocido dirigente estudiantil, Giorgio Jackson (2013), fueron desde mejorar las becas de alimentación hasta acabar con la herencia educacional de la dictadura, pasando por un sinfín de reivindicaciones puntuales como reparar la infraestructura educacional dañada en el último terremoto o generar mecanismos para facilitar la permanencia de estudiantes de los sectores populares en la universidad. No obstante, como ya se adelantó, las reivindicaciones más transversales, las que más apoyo concitaron, fueron tres: democratizar los gobiernos universitarios, poner fin al lucro en la educación superior y obtener gratuidad para el estudiantado. Sobre estas tres demandas se añadirán algunas palabras.

Durante todas las fases del movimiento, pero con especial intensidad en aquellas donde los manifestantes más politizados poseen más prominencia (su ascenso y declive), la demanda por democratizar la universidad tuvo especial resonancia. Esta exigencia presentó dos variantes: que se permitiera al estudiantado participar en los gobiernos universitarios -sea en las instancias de decisión y/o en la elección de autoridades- y que fueran admitidas las organizaciones estudiantiles en algunas instituciones privadas donde estaban prohibidas (CONFECH, 2011b). Una demanda que, además de expresar disconformidad con el modo como se conducian las instituciones de educación superior, transmitía la convicción de que la participación estudiantil facilitaría la resolución de conflictos al interior de la comunidad universitaria.

Es oportuno recordar que la demanda por democratizar la universidad posee una vasta historia en América Latina, pues fue ella la que dio su sello a un movimiento estudiantil que en 2018 conmemoró su primer centenario, el movimiento argentino de 1918, y fue ella la protagonista de sendos movimientos estudiantiles en Chile durante la década de 1960, los mismos que terminaron por darle participación efectiva al estudiantado en los gobiernos universitarios ${ }^{5}$. Se debe sopesar, además, que la supresión de dicha conquista, así como la prohibición de prácticamente todas las organizaciones estudiantiles, fue una de las primeras medidas de la dictadura militar. Y es necesario considerar, igualmente, que el primer gran movimiento estudiantil en democracia, el de 1997, consiguió participación efectiva de los estudiantes en el gobierno universitario de una de las principales universidades del país, la Universidad de Chile. Por todo esto es que se sostiene que así como democratizar las universidades no era una demanda novedosa, tampoco era una exigencia antojadiza o extemporánea.

5- En el tercer cuarto del siglo XX esta demanda también fue central en los movimientos de Uruguay en 1958 (MARKARIAN; JUNG; WSCHEBOR, 2008), de Brasil en 1962 (CUNHA, 1983) y de México en 1971 (RIVAS ONTIVEROS, 2007). 
La demanda por poner fin al lucro en las universidades, en tanto, fue consolidándose en los momentos de mayor intensidad del movimiento y tenía que ver con hacer cumplir la Ley n.18.962 -artículo 30- (CHILE, 1990), que prohibía a las instituciones de educación superior funcionar como cualquier otra actividad económica, es decir, procurando obtener ganancias para sus dueños. Exigencia que venía siendo formulada en diferentes tonos al menos desde el movimiento de 2006 y que se anclaba en la constatación de que aquellas universidades que lucraban eran las que entregaban una educación comparativamente más deficiente y, por ende, eran las que con más urgencia necesitaban que se les inyectaran recursos para mejorar su calidad -y no que se les retiraran en forma de ganancias o lucro-. Diagnóstico que se agravaba al considerar que estas instituciones acogían a los estudiantes con una base educacional más descuidada y que, por lo mismo, necesitaban con urgencia una educación superior mucho más prolija. Para dejar bien asentada esta idea se subraya que la sensación imperante entre el estudiantado era que había quienes estaban haciendo negocio con la ilusión de los sectores más desprotegidos de la población y que, por cuidar de sus utilidades, estaban traicionando su misión educacional (CONFECH, 2011a).

$\mathrm{Al}$ poner al lucro en el centro de los agravios el estudiantado no solo estaba cuestionando al modelo neoliberal de educación superior, también estaba denunciando la existencia de dos circuitos universitarios con disímiles calidades. Uno para los sectores dirigentes, los que gozaban de las mejores condiciones para estudiar y no tenían dificultades para costear su educación superior, y otro para los sectores populares, a los que solo les tocaba acceder a ese mínimo educacional que garantiza el Estado y que solo pueden ingresar a la universidad luego de hipotecar su futuro a la banca privada. Vista en estos términos la ecuación, para los estudiantes, era simple: para algunos, los menos, tocaba lo mejor, mientras para los demás, los más, restaba lo que alcanzaban a pagar con su dinero o, más ajustadamente, con su capacidad de endeudamiento (VALLEJO, 2012). Lógica donde la educación, como sostuviera públicamente el Presidente de la República en medio del conflicto, funcionaba como cualquier otro bien de consumo (EMOL, 2011).

La demanda por gratuidad, en tanto, también fue ganando fuerza a medida que crecía el movimiento. Con ella los manifestantes buscaban poner atajo a las dificultades que desde 1981 tenían los estudiantes (y sus familias) para costear sus estudios superiores. Una situación que se fue agudizando a medida que se consolidaba la política neoliberal de financiamiento de la educación superior y que en 2011 se tornó intolerable con la onerosa carga económica que significaba pagar el Crédito con Aval del Estado (CONFECH, 2011c). Un escenario que se volvía más dramático aún para aquellos estudiantes que debían desertar, pues adquirían una pesada deuda y se quedaban sin título. Pese a que no existen estadísticas consolidadas sobre la deserción universitaria, ésta se infiere significativa puesto que, según datos recientes, solo en el primer año de estudios el abandono estudiantil bordea los treinta puntos porcentuales -siendo más acentuado aún entre el estudiantado con menores recursos económicos- (BRUNNER, 2015).

Aunque los manifestantes no consiguieron aunar fuerzas en torno a una fórmula para conseguir la gratuidad ${ }^{6}$, lo que sí estaba claro era la intencionalidad de la misma.

6- Luego del movimiento aparecieron diferentes propuestas para implementar la gratuidad. Entre ellas destaca la que entiende que el Estado debería financiar la universidad para todos los estudiantes con recursos obtenidos de un impuesto especial a los graduados (ATRIA, 2013). 
Se deseaba garantizar el acceso, permanencia y egreso de todos los estudiantes para así evitar que, por falta de recursos, los estudiantes talentosos, como refirieran en más de alguna oportunidad los dirigentes estudiantiles, quedaran al margen de la educación superior. Un trasfondo que ese 2011 también estaba presente en otras reivindicaciones estudiantiles como las referidas a aumentar el financiamiento público a las universidades estatales, mejorar las condiciones de transporte y alimentación estudiantil, diversificar los procedimientos de ingreso a las universidades y apoyar mediante mecanismos especiales -de nivelación, mantención y titulación- a los estudiantes de sectores medios y populares. Todas demandas que, pese a su similitud con otras presentes en diversos movimientos que han sacudido a los países de la región en los últimos cien años, adquieren connotaciones diferentes según el contexto en que se enmarcan. Por ejemplo, mientras el movimiento estudiantil verificado en la Universidad Nacional Autónoma de México en 1999-2000 defendió el carácter gratuito de la universidad ante las arremetidas de las políticas neoliberales (MUÑOZ TAMAYO, 2011), como ocurrió también en Colombia en 2011, en el caso aquí estudiado los estudiantes persiguieron arrebatarle al neoliberalismo una de sus conquistas más preciadas: la mercantilización de la educación superior.

\section{El trasfondo educacional de la crítica estudiantil al neoliberalismo}

Hemos llegado al corazón del artículo, al momento donde podremos apreciar el trasfondo educacional de la crítica estudiantil al neoliberalismo. Objetivo que se logrará analizando integralmente, con la ayuda de algunos de sus principales analistas/ intérpretes, a las tres demandas estudiantiles recién caracterizadas. La primera lectura posible a estas exigencias, quizá la más evidente, denota que con ellas se buscaba mejorar las condiciones de estudio para los jóvenes con una situación económica comparativamente más desmedrada. Se perseguía, visto desde una perspectiva más amplia, que los estudiantes pudieran desarrollar su labor sin esa onerosa carga/deuda que los distraía de sus objetivos educacionales y que muchas veces los obligaba a desertar. Es por esto que el estudiantado demandaba gratuidad y es por esto, para defender esta postura, que buscaba tener mayor protagonismo en los gobiernos universitarios.

Una segunda lectura a estas demandas, especialmente a las de poner fin al lucro y lograr gratuidad, aprecia que ellas pretendían sacar a la educación superior de la órbita del libre mercado para instalarla en otra lógica. ¿Cuál lógica? Aunque ella ni siquiera era evidente para sus protagonistas, sí se sabía que había que abandonar esa concepción que entiende a la educación como negocio y que el camino no era replicar las experiencias exitosas bajo el marco neoliberal, es decir, mejorar dicho marco. Es sobre estas premisas que se bosquejarán los pilares de la lógica educacional que en 2011 se defendía.

La postura que el estudiantado defendió, como advierte Carlos Ruiz Schneider (2013), entendía a la educación como un derecho y concebía al Estado como el encargado de garantizarlo. Por esto, a diferencia de la matriz neoliberal en que el Estado solo alienta el accionar de los privados y asegura un mínimo educacional a quienes no 
pueden participar por sus propios medios del mercado educacional, en esta otra matriz el Estado debía garantizar una educación de calidad en los diferentes niveles del sistema en función de los intereses, necesidades y méritos de los estudiantes. Una lógica que implicaba contar con un Estado capaz de orientar al sistema educacional conforme a un proyecto, uno que "[...] no reproduzca las desigualdades" (CONFECH, 2011c, p. 16), y no, como todavía ocurre, que se resigna a ver como el sistema educacional refleja la estratificación de la sociedad. Una misión que no renuncia a la búsqueda del bien común o, mejor dicho, que vincula estrechamente educación, bien común y justicia social (PUIGGRÓS, 2018).

Conforme interpretaciones sostenidas por Alberto Mayol (2012), uno de los intelectuales que acompañó más de cerca al movimiento, los manifestantes buscaban asegurar la calidad de las instituciones con incentivos basados en la equidad, no en la competencia. Lo que significa, por ejemplo, que cuando una universidad fuera evaluada como deficiente en comparación a las demás, a ésta debería apoyársela con más esmero, con más recursos. No con menos como ocurre hoy, no con estigmas, no con sanciones. Esto implica, igualmente, que la universidad debería hacerse corresponsable del éxito o fracaso escolar de su estudiante, en el sentido que cuando un joven presentara un ritmo de estudio más lento, o evidenciara habilidades diferentes a las comprendidas como deseables, no se le debería castigar, al contrario, debería invertirse más en él, apoyarlo con más decisión y con más recursos. Al final, lo que primaba, era la comprensión de que era de interés del conjunto de la sociedad el tener una población inquieta, reflexiva y comprometida con el bien común. Dicho con otras palabras, se comprendía como beneficioso para todos el que las mejoras en educación no provinieran de la mano "[...] del individualismo, la competencia y el exitismo personal" (VALLEJO, 2012, p. 57) sino de la solidaridad, del que todos ganan cuando gana el otro, del que todos ganan cuando se tiene una población mejor educada (APPLE, 2013).

Conforme lo dicho, ¿qué papel atribuía el estudiantado a la educación en la transformación de la sociedad? A diferencia del estrecho papel que se le reserva en la lógica neoliberal donde se aspira, en lo medular, a que el mercado educacional funcione autónomamente (ATRIA, 2014), en 2011 los manifestantes bosquejaron una postura tentativamente particularizada como liberal ${ }^{7}$. Postura donde se comprendía a la educación como fundamental para combatir los problemas que afectan a la sociedad, entre ellos la desigualdad, y que la concebía como la vía por excelencia para promover a los mejores elementos de la sociedad, a los más esforzados o talentosos, a los puestos de élite o de dirección de la misma. Una posición presente en múltiples aristas del movimiento, pero sobre todo en el subtexto de la persistente crítica estudiantil al sistema de selección universitaria por discriminar según el sector social del estudiante y no por sus méritos o talentos (CONFECH, 2011b). Para ser bien claros en este punto, y espantar de paso los peores fantasmas dejados por algunos totalitarismos del siglo

7- En un eje horizontal se distinguen, con base en aproximaciones como las de Figueroa (2017), Gane (2015), Harnecker (1979) y Puiggrós (2018), cuatro grandes lógicas de ordenamiento social en la historia moderna latinoamericana. De izquierda a derecha ellas son: comunista, socialista, liberal y neoliberal. Distinción gruesa pero que sirve para situar la visión estudiantil en la centro-derecha de ese continuum, distinguiéndola tanto de la derecha neoliberal como de las izquierdas socialistas y comunistas. 
XX, no se buscó homogeneizar la provisión de contenidos, ampliar indefinidamente el acceso a la universidad, acabar con las instituciones de educación superior privadas o alterar la estructura social vigente ${ }^{8}$. Se aspiró, más modestamente, a que la educación ubicara a los individuos en la estructura social según sus méritos, en los términos que lo entienden también Bovens y Anchrit (2017), tornando de paso más porosa la frontera entre los diversos sectores sociales.

Por esto, a diferencia de la visión neoliberal que comprende que los mejores son al mismo tiempo los que tienen más recursos económicos (MAYOL, 2012), visión que por lo tanto normaliza la existencia de circuitos educacionales con disímiles calidades, en la concepción liberal de los manifestantes chilenos en 2011 “[...] los talentos están distribuidos de manera uniforme en toda la población” (CONFECH, 2011b, p. 5) y, por lo mismo, los diferentes sectores sociales debían estar proporcionalmente representados en la universidad. Razonamiento que tornaba impostergable, como bien expresó la dirigenta estudiantil Camila Sepúlveda, que se desvinculara dinero y educación (SEPÚLVEDA, 2014). Significaba, con otras palabras, que el poder adquisitivo del estudiante no debía determinar la educación que éste recibía, que las preocupaciones de la juventud no debían ser las deudas contraídas y que la agenda de las instituciones educacionales no debía estar capturada por los asuntos financieros.

Para despejar cualquier posible mal entendido, los manifestantes no buscaron que la educación fuera una instancia de transformación social abrupta, tampoco pusieron a la universidad en un pedestal sagrado que exigía que se sacrificaran todas las otras áreas de interés común para poder costearla. Se perseguía, más bien, que el criterio de selección para participar en este nivel educacional fuera consensuado colectivamente (los méritos), no condicionado a lo económico. Y es que en la visión de los manifestantes un sistema educacional regido por criterios culturales no solo era diametralmente distinto al que todavía hoy domina, era también un prerrequisito indispensable para terminar con una de sus funciones cardinales: ser uno de los dispositivos de legitimación del neoliberalismo. Por esto es que Camila Vallejo, la principal líder del movimiento de 2011, entendía que un triunfo en el ámbito de la educación podía abrir paso a otras reivindicaciones sociales que apuntaran “[...] a desmantelar poco a poco el modelo neoliberal anquilosado en Chile durante treinta años” (VALLEJO, 2012, p. 9).

No todos los estudiantes movilizados compartieron el trasfondo liberal aquí atribuido a las demandas de 2011, pues algunos se vincularon al movimiento con pretensiones neoliberales -anhelando solamente un alivio para sus maltrechas economías familiares-, mientras otros lo hicieron bajo horizontes socialistas - pretendiendo reorganizar la estructura social y, en algunos casos, inclusive acabar con el capitalismo-. Pero se cree interpretar bien cuando se afirma que la mayoría del estudiantado, así como sus dirigentes más visibles, no pidió profundizar los derroteros neoliberales, ni revolucionar la sociedad, exigió, más bien, la puesta en práctica de principios liberales. Aquella concepción que, conforme informan tanto las críticas como las demandas estudiantiles,

8- Tanto la demanda por transformar la estructura social como por ampliar el acceso a la universidad son leídas como exigencias que cobran sentido en idearios socialistas. Concepciones que, aunque no hayan sido hegemónicas en este tipo de movimientos, han tenido presencia en diferentes levantamientos estudiantiles como los de Brasil en la década de 1960 (MOTTA, 2014). 
entendía a la educación como derecho, otorgaba al Estado un papel protagónico en su satisfacción, ponía al bien común como horizonte y confiaba en que eran la equidad y la solidaridad -por sobre la competencia- las directrices que debían dar sentido a la lógica educacional que podría reemplazar al neoliberalismo.

\section{Conclusiones}

Aunque a corto plazo el movimiento no obtuvo conquistas tangibles, a mediano plazo sí las consiguió: se bajó la tasa de interés de los créditos educacionales de la banca privada, se derogó la legislación que impedía la participación del estudiantado en los gobiernos universitarios, se creó una Superintendencia de Educación para fiscalizar el cumplimiento de las normativas vigentes y se implementaron becas completas de arancel para los estudiantes de los quintiles más pobres (BACHELET, 2016). No obstante, nada se avanzó en acabar con el lucro en la educación superior y, por lo mismo, nada en abandonar la lógica neoliberal. Sus logros concretos, paradójicamente, profundizaron lo que se venía haciendo. Por esto, aunque es imposible desmerecer la importancia de estas conquistas para los estudiantes directamente beneficiados, miradas en conjunto ellas no dejan de ser un triunfo para esa parte de los sectores dirigentes que deseaba perpetuar la estrategia imperante (PLEYERS, 2018).

Pero subvertir el orden vigente no es una vara sensata para evaluar si un movimiento resulta o no victorioso, sobre todo si se concuerda con la interpretación que abre este artículo referida a que varias décadas de hegemonía neoliberal no pasan en vano. Y es que pretender modificar en unos meses todo un ordenamiento social supera las posibilidades de cualquier movimiento, independientemente de la energía que pueda desplegar en las calles. Lo que se debe resaltar, más bien, es que el estudiantado logró cuestionar la hegemonía del neoliberalismo para sacarlo de su pedestal de pensamiento único y aterrizarlo a su condición de ser una alternativa más para organizar la sociedad. ¿Qué quiere decir esto? Que para muchos, desde entonces, el neoliberalismo ya no es el único ordenamiento social, tampoco el mejor, ni siquiera el más eficiente. Parafraseando a Andrés Bernasconi (2015), el movimiento consiguió que se comprendiera al neoliberalismo como una construcción ideacional con supuestos, estrategias y metas que cumple medianamente bien, pero que no sirve para resolver asuntos que escapan a sus intereses como lo son, precisamente, aquellos por los que pelearon los estudiantes. Asuntos que, como enseñan los especialistas del Programa de las Naciones Unidas para el Desarrollo (PNUD, 2015), parte de la sociedad chilena también ha venido asumiendo como suyos, sobre todo aquellos referidos a contrarrestar la creciente desigualdad.

Fue gracias al movimiento estudiantil de 2011, entonces, que en Chile se ha abierto la posibilidad de preguntarse por asuntos que antes ni siquiera hubieran podido formularse, por ejemplo: ¿Debemos limitarnos a intentar terminar con la pobreza o podemos también aspirar a contener la creciente desigualdad? ¿Aporta al bien común que existan universidades cada vez más segmentadas en función de la capacidad de pago de los estudiantes? ¿Es conveniente que el estudiantado enfrente su educación condicionado por las deudas que está contrayendo? Preguntas que, como se ha podido 
apreciar, los manifestantes respondieron con una concepción educacional diferente a la que impera desde la década de 1980. ¿Cuál concepción? Una que se inscribe en el amplio registro del pensamiento liberal, que tiene a la solidaridad, el bien común y la equidad como pilares estructurantes, que concibe al Estado con un papel activo en la educación (apoyando decididamente tanto a las instituciones como a los estudiantes con más dificultades) y que comprende que deben ser los criterios culturales, como los méritos, los que han de imponerse a la hora de promover a los estudiantes a los diferentes niveles del sistema educacional, más aún a la universidad.

Pero el que se haya puesto en duda la hegemonía del libre mercado, y el que se haya abierto una brecha a través de la cual hoy es posible pensar otro tipo de educación, no significa que el neoliberalismo esté condenado al olvido ni que el liberalismo defendido por los estudiantes llegue a imponerse en el corto o mediano plazo. Significa que se han abierto debates, antes impensables, sobre asuntos que ciertamente trascienden la frontera nacional, por ejemplo, aquel referido a qué tan posible es transitar desde una lógica educacional neoliberal a otra diferente (liberal según los términos que aquí se plantean). Debate donde están quienes subrayan las dificultades que tiene esta empresa por lo arraigado que está el neoliberalismo en el conjunto de la población (DONOSODÍAZ, 2016), quienes señalan que es posible pasar de una a otra lógica haciendo pequeños ajustes en sectores claves del sistema educacional como los relativos al financiamiento (ATRIA, 2014) y quienes entienden que, si bien no hay que renunciar a lucha educacional, los esfuerzos se deben enfocar en tensionar al sistema político para obligarlo a sancionar una nueva constitución que abra paso, ahora sí, a transformaciones sustantivas en la educación (GARCÉS, 2012).

Recapitulando, el movimiento estudiantil chileno de 2011 fue, en más de alguna medida, esa protesta que en 1981, cuando la dictadura imponía en la universidad la lógica neoliberal, no pudo darse porque todas las energías estudiantiles estaban enfocadas en acabar con el régimen autoritario. Hoy, después de varias décadas de expulsados los militares del poder, la movilización estudiantil nos recuerda, entre otros aspectos, que el modelo universitario neoliberal solo es uno más, lo que significa que es posible, y legítimo, comenzar a pensar una universidad regida bajo otros preceptos, donde la educación no apunte a ser, simplemente, un bien de consumo.

\section{Referencias}

APPLE, Michael. Can education change society? New York: Routledge, 2013.

ATRIA, Fernando. Derechos sociales y educación: un nuevo paradigma de lo público. Santiago de Chile : Lom, 2014.

ATRIA, Fernando. Sobre la 'gratuidad' de la educación. In: GARCÍA HUIDOBR0, Juan Eduardo; FALABELLA, Alejandra (Ed.). Los fines de la educación: seminarios sobre gratuidad, lucro, justicia y democracia. Santiago de Chile: CEPPE, 2013. p. 57-80. 
BACHELET, Michelle. Mensaje de S.E. la Presidenta de la República con el que inicia un Proyecto de Ley de Educación superior. In: CHILE. Proyecto de ley de educación superior, Michelle Bachelet y otros de 4 jul. 2016. Santiago de Chile: [s. n.], 2016. Disponible en: <https://www.24horas.cl/incoming/article2065068. ece/BINARY/Proyecto\%20de\%20Ley\%20Reforma\%20Educaci\%C3\%B3n\%20Superior>. Acceso en: 28 febr. 2019.

BELLEl, Cristian. El gran experimento: mercado y privatización de la educación chilena. Santiago de Chile: Lom, 2015.

BERNASCONI, Andrés. Introducción. In: BERNASCONI, Andrés (Ed.). La educación superior en Chile: transformación, desarrollo y crisis. Santiago de Chile: PUC, 2015. p. 7-18.

BOVENS, Mark; ANCHRIT, Wille. Diploma democracy: the rise of political meritocracy. New York: Oxford University Press, 2017.

BRUNNER, José Joaquín. Medio siglo de transformaciones de la educación superior chilena. In: BERNASCONI, Andrés (Ed.). La educación superior en Chile: transformación, desarrollo y crisis. Santiago de Chile: PUC, 2015. p. 21-107.

CHILE. Ministerio de Educación Pública. Ley 18.962. Ley orgánica constitucional de enseñanza, 07 de marzo de 1990. Santiago de Chile: Ministerio de Educación Pública, 1990. Disponible en: <https://www. leychile.cl/N?i=30330\&f=2005-09-27\&p=>. Acceso en: 28 febr. 2019.

CONFECH. Confederación de Estudiantes de Chile. Bases para un sistema gratuito de educación: demanda estudiantil. Valparaíso: [s. n.], 2011c. Documento emanado por la CONFECH modificado por la Federación de Estudiantes de la Universidad de Valparaíso, 8 sept. 2011. Disponible en: <http://movimientoestudiantil.cl/ wp-content/uploads/2015/12/2011-09-01-bases-para-un-sistema-gratuito.pdf>. Acceso en: 28 febr. 2019.

CONFECH. Confederación de Estudiantes de Chile. Carta abierta al señor Joaquín Lavín Infante. Santiago de Chile: [s. n.], 2011a. Disponible en: <http://movimientoestudiantil.cl/wp-content/uploads/2015/12/201105-26-Carta-Ministro-Lavin.pdf>. Acceso en: 28 febr. 2019.

CONFECH. Confederación de Estudiantes de Chile. Reforma educación superior: ejes principales de las demandas estudiantiles. Santiago de Chile: [s. n.], 2011b. Disponible en: <http://movimientoestudiantil.cl/wpcontent/uploads/2015/12/2011-06-14-presentacion-confech-demandas.pdf>. Acceso en: 28 febr. 2019.

CUNHA, Luiz Antônio. A universidade crítica: 0 ensino superior na república populista. Rio de Janeiro: Francisco Alves, 1983.

DELLA PORTA, Donatella; DIANI, Mario. Introtuction: The field of social movements studies. In: DELLA PORTA, Donatella; DIANI, Mario (Ed.). The Oxford handbook of social movements. Oxford: Oxford University Press, 2015. p. 1-27.

DONOSO, Sofía. Dinamics of change in Chile: explaining the emergence of the pingüino movement. Journal of Latin American Studies, London, v. 45, n. 1, p. 1-29, 2013. 
DONOSO DÍAZ, Sebastián. Chilean education policy between the student movement of 2011 and reformist debates of 2014. Universum, Talca, v. 31, n. 1, p. 105-121, 2016.

DRISKO, James; MASCH, Tina. Content analysis. New York: Oxford University Press, 2016.

EMOL. Presidente Piñera afirma que la educación "es un bien de consumo". Emol, Santiago de Chile, 11 jul. 2011. Noticias. Disponible en: <https://www.emol.com/noticias/nacional/2011/07/19/493428/ presidente-pinera-afirma-que-la-educacion-es-un-bien-de-consumo.html>. Acceso en: 28 febr. 2019.

FAURÉ, Daniel; MIRANDA, Esteban. Prólogo. In: Fauré, Daniel; Miranda, Esteban (Ed.). 4 de agosto: testimonios de una revuelta popular. Santiago de Chile: Universidad de Chile, 2016. p. 13-21.

FIGUEROA, Francisco. Llegamos para quedarnos: crónicas de la revuelta estudiantil. Santiago de Chile: Lom, 2013.

FIGUEROA, Víctor. Latin American communism. In: NAIMARK, Norman; PONS, Silvio; QUINN-JUDGE, Sophie (Ed.). The Cambridge history of communism. v. Il. Cambridge: Cambridge University Press, 2017. p. 388-413.

GANE, Nicholas. Trajectories of liberalism and neoliberalism. Theory, Culture \& Society, London, v. 32, n. 1, p. 133-144, 2015.

GARCÉS, Mario. El despertar de la sociedad: Ios movimientos sociales en América Latina y el Caribe. Santiago de Chile: Lom, 2012.

GARCÍA HUIDOBR0, Juan Eduardo; FALABELLA, Alejandra. Diálogos sobre los fines de la educación. In: GARCÍA HUIDOBRO, Juan Eduardo; FALABELLA, Alejandra (Ed.). Los fines de la educación: seminarios sobre gratuidad, lucro, justicia y democracia. Santiago de Chile: CEPPE, 2013. p. 7-16.

GARCÍA MONGE, Diego; ISLA MADARIAGA, José; TORO BLANCO, Pablo. Los muchachos de antes: historias de la FECH 1973-1988. Santiago de Chile: UAH, 2006.

GARRETÓN, Manuel Antonio. Neoliberalismo corregido y progresismo limitado: los gobiernos de la concertación en Chile, 1990-2010. Santiago de Chile: ARCIS-CLACSO, 2012.

HARNECKER, Marta. Socialismo y comunismo. Madrid: Akal, 1979.

JACKSON, Giorgio. El país que soñamos. Santiago de Chile: Random House, 2013.

LAVÍN da las claves para la reforma de la educación superior. In: CHILE. Ministerio de Educación. Anticipos sobre política de educación superior Santiago de Chile: SME, 2011. Dossier Mayo 20, 2011. p. 1-13. Dispnible en: <https://www.archivochile.com/Chile_actual/01_mse/2/MSE2_0001.pdf>. Acceso en: 28 febr. 2019.

MARKARIAN, Vania; JUNG, María Eugenia; WSCHEBOR, Isabel. 1958 el cogobierno autonómico. Montevideo: Archivo General Udelar, 2008. 
MAYOL, Alberto. No al lucro: de la crisis del modelo a la nueva era política. Santiago de Chile: Random House, 2012.

MOTTA, Rodrigo Patto Sá. As universidades e o regime militar. Rio de Janeiro: Zahar, 2014.

MUÑOZ TAMAYO, Víctor. Generaciones: juventud universitaria e izquierdas políticas en Chile y México (U. de Chile - UNAM 1984-2006). Santiago de Chile: Lom, 2011.

OCDE. Organización para la Cooperación y el Desarrollo Económico. Revisión de políticas nacionales de educación: la educación superior en Chile. Santiago de Chile: OCDE/BIRD/BM/MINEDUC, 2009.

PLEYERS, Geoffrey. Movimientos sociales en el siglo XXI. Buenos Aires: Clacso, 2018.

PNUD. Programa de Naciones Unidas para el Desarrollo. Desarrollo humano en Chile: los tiempos de la politización, 2015. Santiago de Chile: PNUD, 2015.

PUIGGGRÓS, Adriana. Neoliberalism and education in the Americas. New York: Routledge, 2018.

REYES, Carolina; VALLEJO, Javiera. Los días que avanzaron años: el movimiento estudiantil 2011 desde la perspectiva de sus dirigentes. Santiago de Chile: Ceibo, 2013.

RIESCO, Manuel. Parto de un siglo: una mirada al mundo desde la izquierda de América Latina. Santiago de Chile: Cenda/Usach, 2012.

RIVAS ONTIVEROS, José. La izquierda estudiantil en la UNAM: organizaciones, movilizaciones y liderazgos (1958-1972), México, DF: Porrúa/UNAM, 2007.

RUIZ ENCINA, Carlos. De nuevo la sociedad. Santiago de Chile: Lom, 2015.

RUIZ SCHNEIDER, Carlos. Educación y democracia: aportes para el cambio desde la demanda estudiantil. In: GARCÍA HUIDOBRO, Juan Eduardo; FALABELLA, Alejandra (Ed.). Los fines de la educación: seminarios sobre gratuidad, lucro, justicia y democracia. Santiago de Chile: CEPPE, 2013. p. 139-162.

SALAZAR, Gabriel; PINTO, Julio. Historia contemporánea de Chile V: niñez y juventud. Santiago de Chile: Lom, 2014.

SALAZAR, José; LEIHY, Peodair. El largo viaje: Ios esquemas de coordinación de la educación superior chilena en perspectiva. Archivos Analíticos de Políticas Públicas, Tempe, v. 4, n. 25, p. 1-26, 2017.

SALAZAR, José; LEIHY, Peodair. El manual invisible: tres décadas de políticas de educación superior en Chile (1980-2010). Archivos Analíticos de Políticas Públicas, Tempe, v. 34, n. 21, p. 1-35, 2013.

SEPÚLVEDA, Claudia. Haremos los cambios a los que los adultos no se atrevieron. In: ANDINO, Rubén (Ed.). La rebelión estudiantil en Chile. Santiago de Chile: Ocean Sur, 2014. p. 17-18. 
THIELEMANN, Luís. La anomalía social de la transición: movimiento estudiantil e izquierda universitaria en el Chile de los noventa (1987-2000). Santiago de Chile: Tiempo Robado, 2016.

TILLY, Charles; TARROW, Sidney. Contentious politics. New York: Oxford University Press, 2015.

VALLEJO, Camila. Podemos cambiar el mundo. Santiago de Chile: Ocean Sur, 2012.

Recibido en: 01.03.2019

Revisado en: 12.08.2019

Aprobado en: 01.10.2019

Andrés Donoso Romo es doctor en Integración de América Latina por la Universidade de São Paulo. Actualmente es investigador del Centro de Estudios Avanzados de la Universidad de Playa Ancha (Chile) y profesor asistente del Instituto de Estudios Avanzados en Educación de la Universidad de Chile. 